\title{
The Effect of Overlapping Area on Ping-Pong Handover in LTE Networks
}

\author{
Kinan Ghanem \\ Department of Computer Eng., \\ AASTMT, Lattakia, Syria \\ kinanghanem@yahoo.co.uk
}

\author{
Haysam Al-Radwan \\ Department of Communications, \\ Tishreen University, Lattakia, \\ Syria haysamradwan@yahoo.com
}

\author{
Ahmad Ahmad \\ Faculty of Information \\ Technology \\ Al-Baath University, Homs Syria \\ ahmad770077@gmail.com
}

\begin{abstract}
Handover (HO) technique in LTE networks suffers from Ping-pong movement. Ping-pong HO can reduce the quality of the mobile user's connection and increases the numbers of handovers which in turn raises the network load and generally degrades the network performance. The work aims to present a novel approach to reduce the undesirable effects of ping-pong $\mathrm{HO}$ in LTE Mobile Networks using timer. The study focused on the ping-ping phenomenon taking into account maintained the dropped calls rates at lowest levels. The optimal timer values are determined based on the width of overlapping area, user velocity and type of eNodeB. Analyzed results showed that the changes of overlapping area directly affect the timer values of the proposed algorithm. Optimal timer value should be selected precisely according to the width of the overlapping area, user velocity and timer value in order to reduce the ping-pong $\mathrm{HO}$.
\end{abstract}

Keywords- Ping-Pong handover, Timer value, LTE, Overlapping area, dropped calls rate.

\section{INTRODUCTION}

Handover is a crucial procedure for maintaining the connection between the mobile users. There are two types of HOs to communicate users between different cells. The first type is called hard HO which is employed in the GSM and LTE mobile networks. The second type is called soft HO which is implemented in IS-95 and 3G mobile networks. LTE is based on OFDMA technology, which is primarily a frequency division process. This means that the user has to actually change into a different set of frequency subcarriers when it hands over between two eNBs, which eliminates the possibility for soft handover. Soft HO has better performance on both link and system level and it has the advantages of smoother transmission and less ping-pong effects. However, it wastes the radio resources and has the disadvantages of complexity, and suffers from ping-pong [1-4].

The ping-pong $\mathrm{HO}$ is a very common phenomenon in the LTE mobile networks, which may cause call dropping and degrading of the HO performance. Moreover, it wastes the limited radio resources because it reserves two connections for a short time. It occurs due to the repeated movement of UE between the source and the target eNB, or high signal fluctuation at the common boundary of the eNBs. Coverage parameters, antenna configuration, users' location area and their movement are the main considerations that can cause the ping pong [5]

Previous works on hard handover led to several algorithms to improve the HO technique in LTE networks. Many studies have been conducted in the area of ping-pong $\mathrm{HO}$ in LTE Networks [6-8]. Two main general methods, hysteresis [6] and TTT (Time To Trigger) with threshold [9-10], have been widely used to solve the Ping-pong HO problem. Previous studies vary from statistical analysis in the literature [11-14] to follow algorithms rely on probability prediction using neural networks in [15] and [16]. In [5], a novel handover algorithm to reduce the ping-pong $\mathrm{HO}$ is presented. However, the effects of overlapping area on the pingpong phenomenon have not been addressed in[5], and Little is known about the effects of the width of overlapping area on ping-pong HO in EUTRA Networks.

The rest of the paper is organized as follows. In section II we present the intra-EUTRA HO procedure. The proposed technique which reduces ping-pong HO based on timer value- is described in section III. The mathematical calculation of the timer value based on the overlapping area is illustrated in section IV. Section V shows the simulation results, also conclusions and future outlook are given in section VI.

\section{LTE INTRA-EUTRA HANDOVER PROCEDURE}

In LTE, the HO decision is made in the eNB without connecting to the MME. The required HO information is exchanged between the eNBs via the X2 interface. According to the $3 \mathrm{GPP}$, the $\mathrm{HO}$ procedure is divided into two parts mainly: the $\mathrm{HO}$ preparation and execution parts and HO completion part. Fig. [1] Shows the intra-EUTRA HO steps and a summary of the HO procedure is summarised in [5]. 


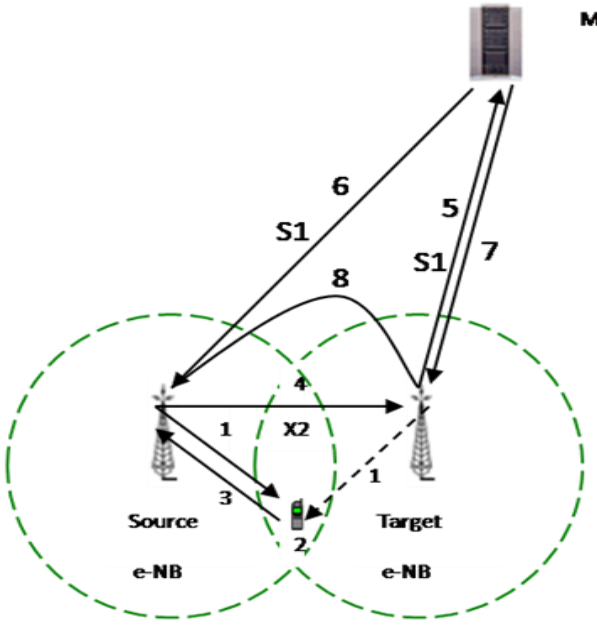

Figure 1: Summary of the different steps of preparation, execution and completion HO process which performs by eNBs. 1) Downlink HO measurements, 2) processing of downlink measurements, 3) uplink reporting, 4) $\mathrm{HO}$ preparation and execution via $\mathrm{x} 2$ interface, 5) path switch request, 6) release the old path, 7) Path switch acknowledgement, 8) Release resources [4, 5].

\section{PING-PONG DETECTION ALGORITHM FOR INTRA LTE HANDOVER}

In the proposed algorithm explained in Fig. 2 a timer is used to delay the $\mathrm{HO}$ completion part from the $\mathrm{HO}$ process.

If the difference between the Signal strength of the target (SS-target) and SS-source always shows that the SStarget is sufficiently stronger than the SS-source after expiring the timer then there is no ping-pong HO. However, if the difference between the SS-target and SSsource does not show that, then the movement is pingpong. In this case, the $\mathrm{HO}$ should be delayed and the old path (MME/SGW-source eNB) should be kept during the ping-pong interval. In another word only the completion part of the $\mathrm{HO}$ procedure can be delayed to avoid the swinging between of the (old and new) paths as it can be seen in (Fig. 2).

The proposed algorithm has 3 phases as explained below. As it appears in fig. 3, the preparation and execution $\mathrm{HO}$ phase means that the new connection between the UE and the target eNB is made but the old $\mathrm{S} 1$ interface is still in use (Blue line in fig. 3). For the HO completion part there is completely new connection path via new S1 interface. It is good to mention that in the completion phase the old $\mathrm{S} 1$ path is released and a new $\mathrm{S} 1$ interface is initiated to be used.

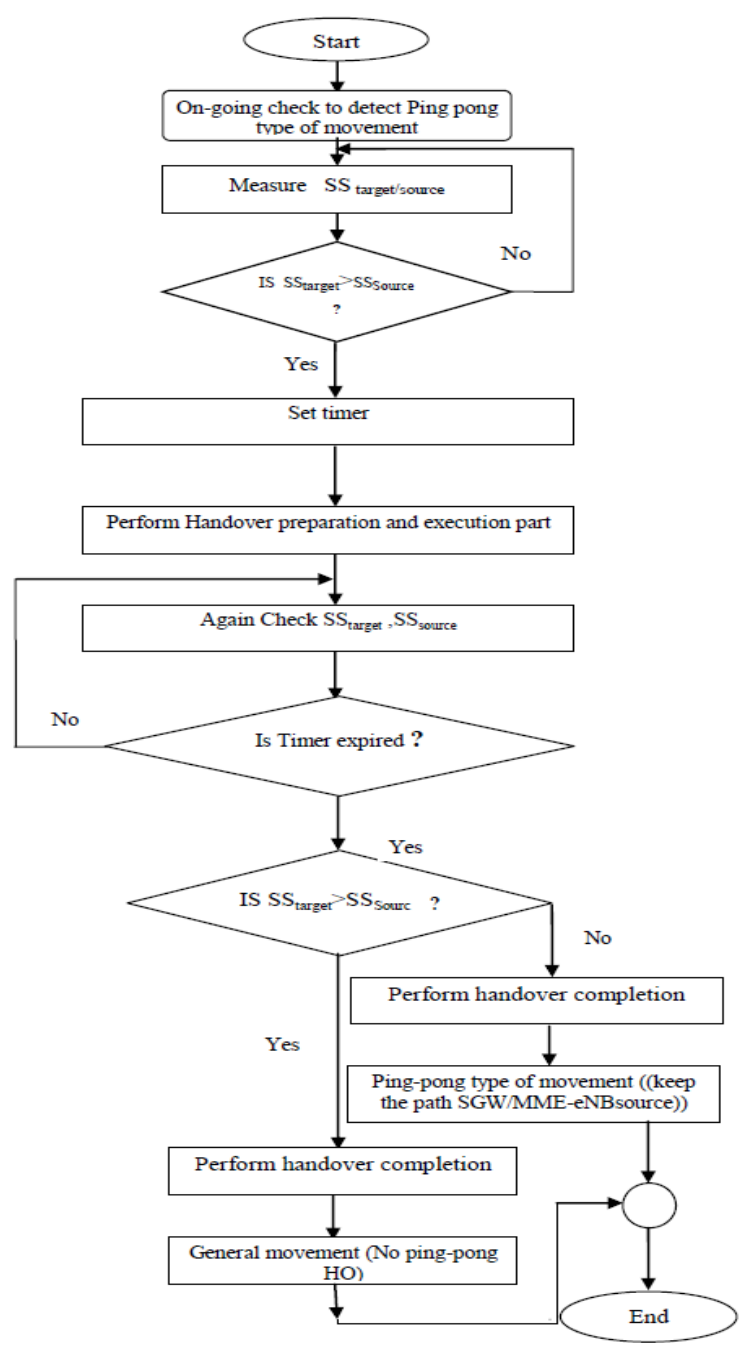

Fig. 2 proposed technique for ping-pong avoidance In Intra E-UTRA

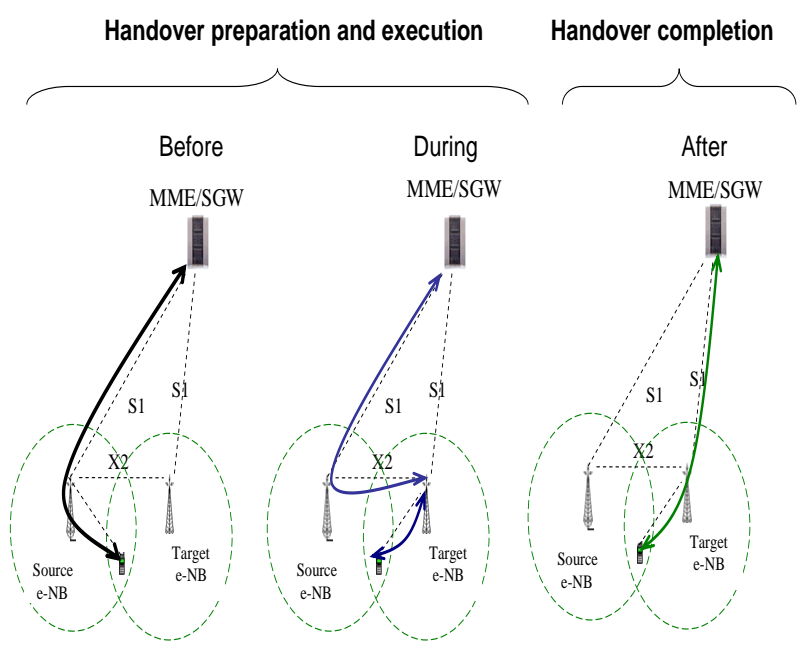

Figure 3: the phases of the proposed algorithm 


\section{ANALYSIS OF THE PROPOSED ALGORITHM}

\section{A. How to define the difference SS (target-source)} mathematically

For each mobile phone in the overlapping area, the average of the standard deviation can give the best $\mathrm{SS}$ (target-source) requires to perform a handover.

$$
\begin{gathered}
\mathrm{SS}_{(\text {soucre })}+\mathrm{SS}_{(\text {source } 2)}+\ldots . \mathrm{SS}_{(\text {source } \mathrm{i}=\mathrm{N})} \\
\text { Average } \text { source }_{i=N}=\sum_{i=1}^{i=N} \frac{S S_{(\text {source })}}{N} \\
\mathrm{SS}(\text { target } 1)+\mathrm{SS}(\text { target } 2)+\ldots \mathrm{SS}(\text { target } \mathrm{i}=\mathrm{N}) \\
\text { Average }{ }_{t \arg e t}=\sum_{i=1}^{i=N} \frac{S S_{(t \arg e t)}}{N} \\
\sum_{i=1}^{i=N} \frac{S S_{(\text {sourcei })}}{N}+\sum_{i=1}^{i=N} \frac{S S_{(t \arg e t)}}{N} \\
2
\end{gathered}
$$

Total average is used to trigger the parameters and perform the HO completion part. The difference between -the received signal strength from the target and the source -(SS(target-source)) of the proposed algorithm should be chosen to be less than Total_Average.

$$
\text { Total_Average }=\frac{\sum_{i=1}^{S S_{(\text {target-source })}} \underset{S}{i=N} \frac{S S_{(\text {source })}}{N}+\sum_{i=1}^{i=N} \frac{S S_{(t \text { arg } e t)}}{N}}{2}
$$

\section{B. Mathematical model for maximum timer value}

Let us suppose that the width of the overlapping area equal to $d$, the distance between the User Equipment (UE) and the source is $r$, whereas the distance between the target and the UE is $r$, and $\mathrm{D}$ is the distance between two eBNs. UE velocity is chosen to be $\mathrm{v}$ and it refers to the speed of the mobile user in $\mathrm{m} / \mathrm{sec}$, $\mathrm{d}$ the active HO area (red colored area in fig. (4)). The red area points to the area that the mobile user should perform the $\mathrm{HO}$ completion before passing it. So due to the velocity of the UE the overall HO time should be completed before the $d / v$, where is $d$ is the distance that the SS(target-source) can match. Moreover, The red area is the area of ping-pong $\mathrm{HO}$ and also it is the area which that may have high probability of dropped calls.

$$
\begin{aligned}
& \mathrm{D}=\mathrm{r}+\mathrm{r}^{\prime}, \mathrm{r}^{\prime} \mathrm{r}^{\prime}=\mathrm{d}^{\prime} \text {, where is } \mathrm{d}^{`} \text { can be calclulated as } \\
& \mathrm{d}^{`}=\mathrm{r}-\mathrm{D}+\mathrm{r}=2 \mathrm{r}-\mathrm{D}
\end{aligned}
$$

The maximum value for delaying the HO should not exceed $d / v$, and the Timer value should be less than $d / v$ $(<\mathrm{d} / \mathrm{v})$.

let us assume that the required time to perform the Ho preparation and execution is $\mathrm{t} 1$, and the required time to perform the handover completion is $\mathrm{t} 2$. The Timer value is set to be $\mathrm{t} 3$, and the interruption time which is the $\mathrm{UE}$ radio connection is dead $\mathrm{t} 4$. The total time to finish the complete $\mathrm{HO}$ procedure is assumed to be $\mathrm{T}$.

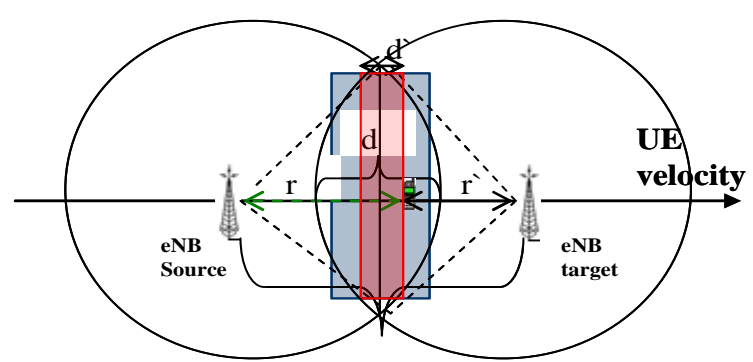

$\mathrm{D}$

Figure 4: Overlapping area ( $2 \mathrm{eNBs}$ ), red area refers to an active $\mathrm{HO}$ area

$$
\mathrm{T}^{\prime}=\mathrm{d} / \mathrm{v}=\mathrm{t} 1+\mathrm{t} 2+\mathrm{t} 3+\mathrm{t} 4
$$

The interruption time, i.e., the time during which the UE radio connection is dead $=\mathrm{t} 1$, T2 HO Execution time, T3 HO trigger until completion.

Let us suppose that $\mathrm{t} 1+\mathrm{t} 2+\mathrm{t} 4=\mathrm{HO}$ overall execution time equal to $100 \mathrm{~ms}$ (It varies from 60 to 100 $\mathrm{ms})$ :

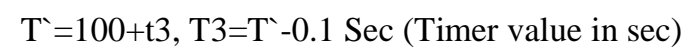

It is good to mention that differs upon the cell size and the cell type. LTE cell sizes may range from femto-cell for indoor/home coverage, to over $30 \mathrm{~km}$ (18.64 miles). However, a typical LTE cell size will be 1 to $5 \mathrm{~km}$ (0.6 - 3 miles), and generally congruent with $2 \mathrm{G} / 3 \mathrm{G}$ cell deployments.

If we suppose that the overlapping area is equal $10 \%$ of the cell size, and the cell diameter in the urban areas equal to 1000 meter than the width of the overlapping area $(\mathrm{d}=100$ meters $)$. Let us suppose that the active HO area equals to $50 \%$ of the overlapping area $\left(\mathrm{d}^{\prime}=\mathrm{d} / 2=50\right.$ meters) then the maximum time for the timer should be $\mathrm{T}=\mathrm{d} / \mathrm{v}$ whereas, $\mathrm{v}$ assigns to the velocity of the UE. If we suppose that the velocity is 25 meter per second than the maximum value is $50 / 25=2 \mathrm{Sec}$. For cell diameter equals to 2400 meters, $d=240$ meters, Then $d^{`}=d / 2=120 \mathrm{~m}$, as a result of that the maximum timer value is $120 / 40=3 \mathrm{Sec}$ (for velocity equals to 40 meters per second).

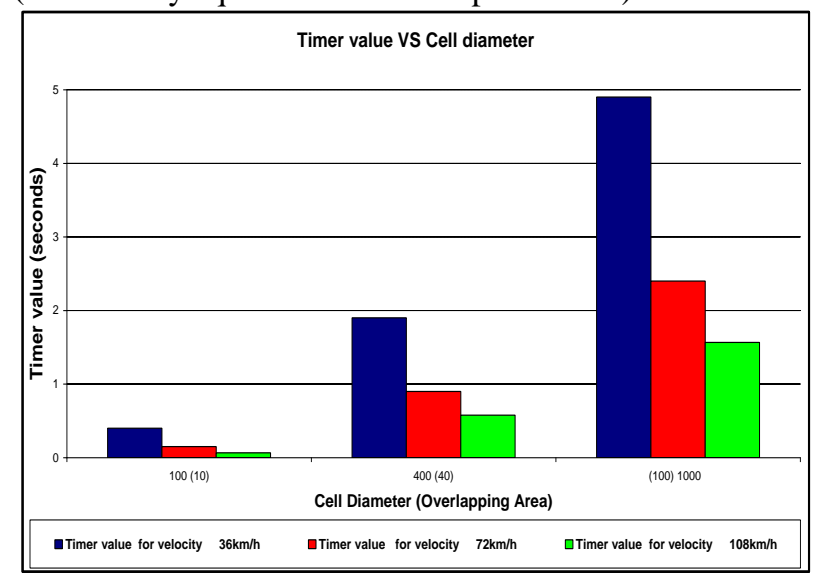


Figure (5) the effects of velocity, cell diameter and overlapping area on the timer values

Increasing the width of overlapping area allows having higher timer value. For different cell diameters -in a real environment-, there will be a different overlapping area as it can be seen in fig. (5). As a result, different Timer values (based on velocity changes) should be applied to complete the HO decision. Rapid changes in the overlapping areas caused by network topology, cell size and the antennas type play significant role in selecting the accurate timer value to reduce the ping-pong $\mathrm{HO}$ effects.

\section{Ping-pong Avoidance Algorithm BASED ON FUZZY LOGIC}

Fuzzy logic technique was previously used to study HO in different mobile networks [17-20] but not to study ping-pong HO. Fuzzy set theory allows a linguistic representation of the control and operational laws of a system in words. The main strength of fuzzy set theory is that it excels in dealing with imprecision. The fuzzy set theory allows the gradual transition from full membership to full non-membership of the set [21-24]. Thus fuzzy set theory is a generalization of classical set theory. In fuzzy set an element is related to a set by a membership function $\mu$. The membership function usually take on a value between 0 and 1 , this means $\mu \rightarrow[0,1]$ where 1 is for full membership, 0 for the null-membership and values in between give the degree of membership.

There are several reasons for using fuzzy control for analyzing the ping-pong HO in E-UTRA networks in this study. The rapid changes in the radio environment require a fast response and better algorithm to follow up these changes. The ping-pong phenomenon is fuzzy since it differs from cell to cell and varies upon radio measurements and dynamic changes in the mobile environment properties. Moreover, the mobile operators are not able to completely control the ping-pong $\mathrm{HO}$ and they use their own experience in reducing it. The pingpong $\mathrm{HO}$ could benefit from the fuzzyfication treatment of the HO input metrics and fuzzy reasoning thereon as it is explained later on this article.

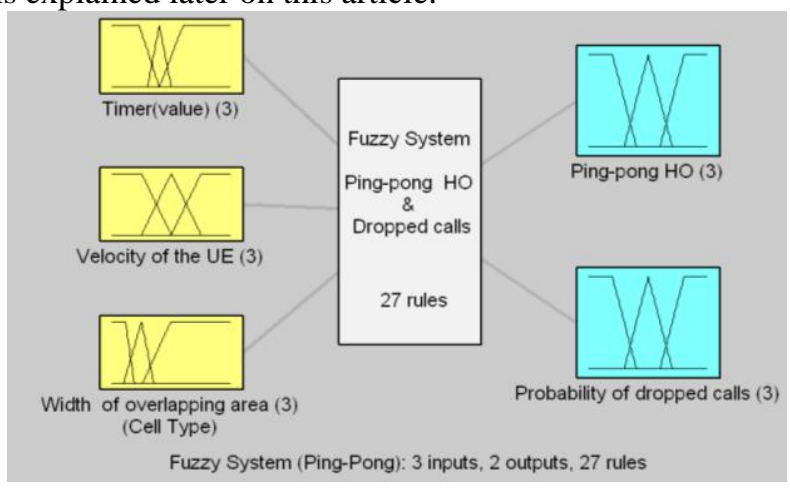

Fig. 6: Fuzzy logic system for Ping-pong Avoidance Algorithm (Matlab)

\section{A. Memberships of Input Parameters}

The input variables for the proposed algorithm are Timer value, velocity of UE and width of overlapping area (Fig. 6). Timer value is assigned the linguistic values Small, Medium, and Big over the range [0,5] Sec. The second input of the proposed algorithm is the velocity of the user, the linguistic values of user velocity is assigned as High, Medium, and Low over the interval [0-100] $\mathrm{km} / \mathrm{hour}$. The third input is the width of overlapping area, the linguistic values of it is assigned as High, Medium, and Low over the range of [0-400] meters. An output parameter refers to the probability of ping-ping $\mathrm{HO}$ which is defined as High, Medium and Low and the corresponding weights are taken to be $1,0.5$ and 0 respectively.

\section{B. Fuzzy inference}

Some fuzzy rules used are presented in table (2):

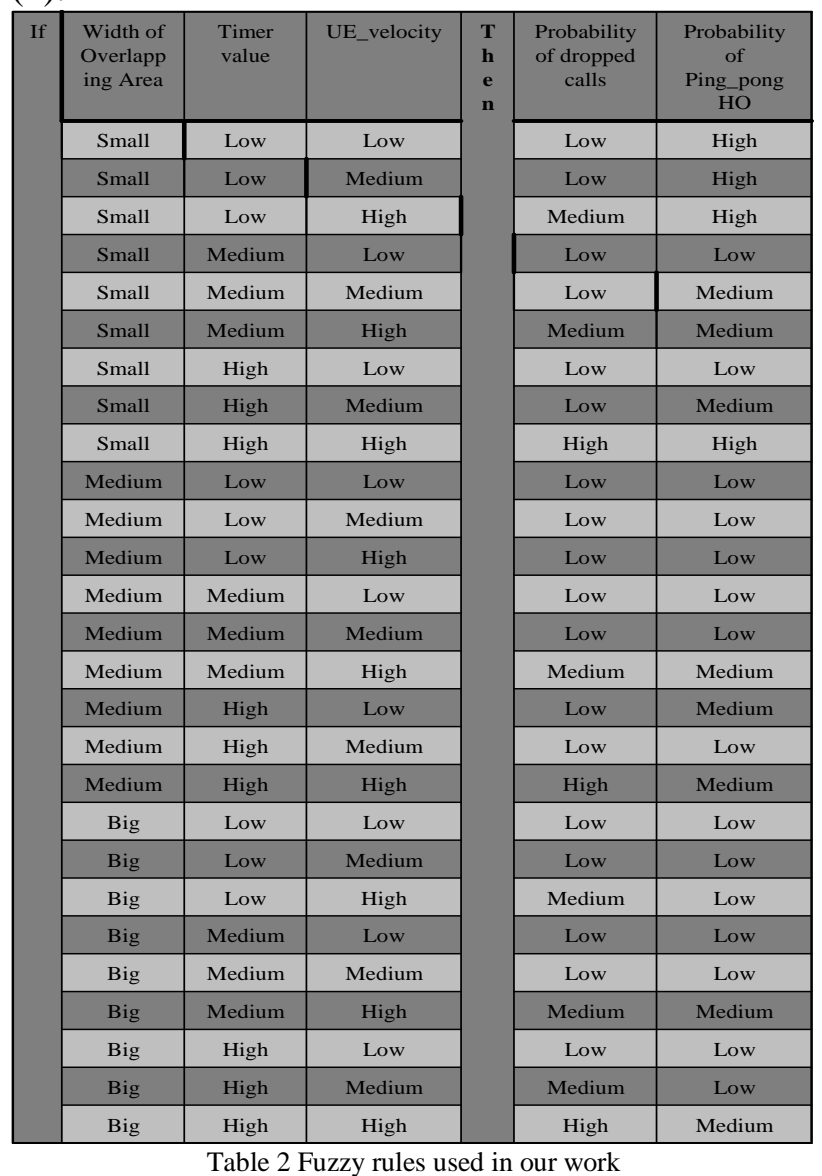

\section{Results and Discussion}

After the membership functions are determined and entered in Matlab Fuzzy Toolbox Membership Function Editor, the rules are selected and written using Matlab rules editor for simulation and evaluation. In the simulation different parameters are chosen according to the 3GPP specifications and recommendations. Moreover, different velocities are selected to study the 
effect of overlapping area on the timer value for different user speeds.

The effects of the inputs functions on the output (probability of ping-pong HO) were analyzed individually and results are shown in figures 7 and 8, respectively (using Matlab Fuzzy Toolbox). Fig. 7 shows that the probability of ping-pong $\mathrm{HO}$ was efficiently reduced at high timer values i.e. more than $1 \mathrm{sec}$. Similarly, Fig. 8 illustrates that a timer value higher than 1.5 seconds decreases the probability of dropped calls rate to the lowest levels.

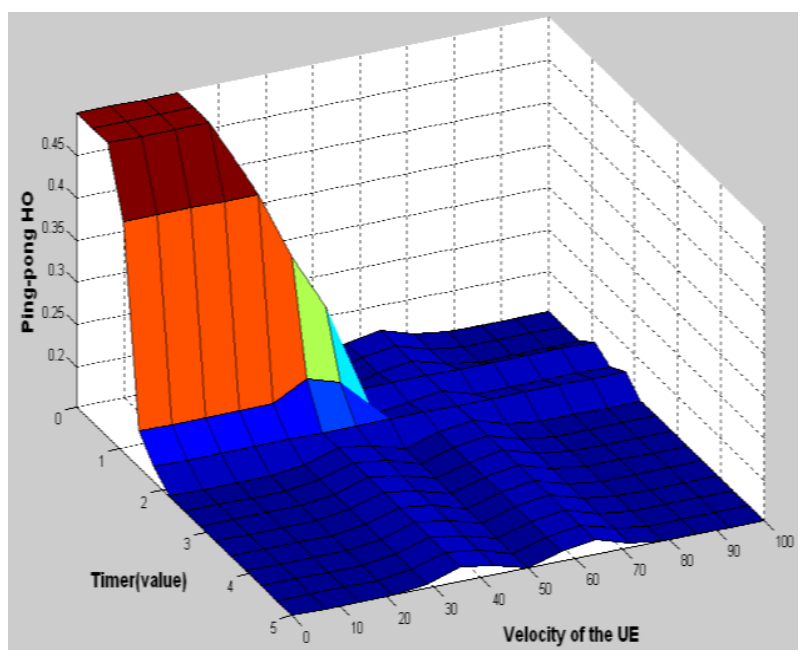

Fig. 7 Surface analysis between time value and UE velocity

Fig. 8 Surface analysis for dropped calls rate ( time value \& UE velocity

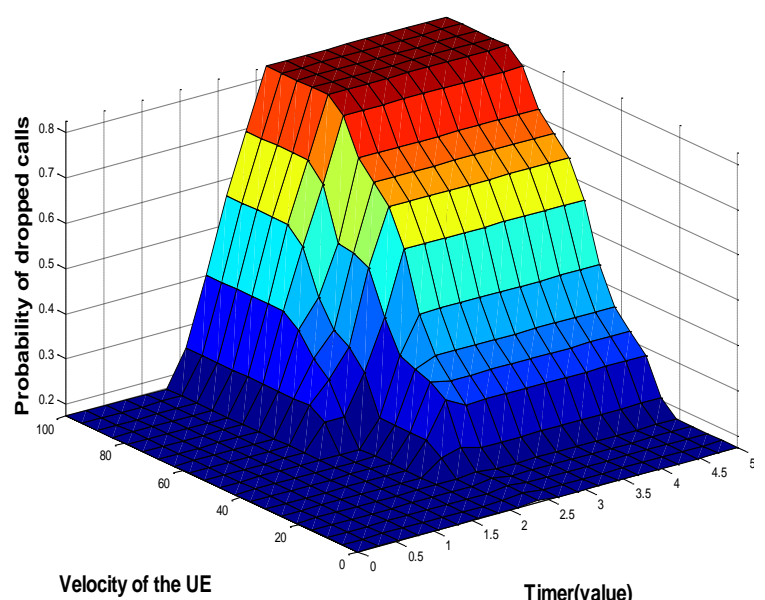

Figures (7-8) show the effects of timer value and UE velocity on the probability of dropped calls and pingpong HO. Results illustrate that the Timer values should not exceed $1.5 \mathrm{sec}$ to keep the probability of dropped calls at lowest rates and reduce the ping-pong $\mathrm{HO}$ at the same time.

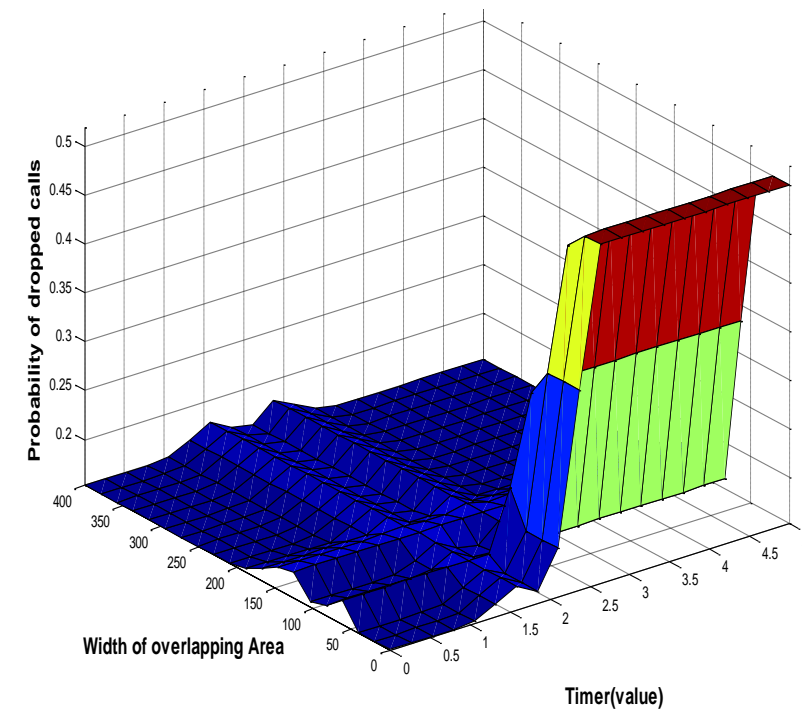

D. The effects of overlapping area on the probability of dropped calls and ping-pong $\mathrm{HO}$

Figures (9-10) show the effects of overlapping area on the probability of dropped calls and ping-pong HO. Results illustrate that the Timer values should not exceed $1.5 \mathrm{sec}$ to keep the probability of dropped calls at lowest rates and reduce the ping-pong $\mathrm{HO}$ at the same time. For high speed user velocities the timer value should be kept less than $1 \mathrm{sec}$ to maintain the dropped calls rates very low (Fig. 11).

A precise tradeoff between the timer value and the width of the overlapping area should be made to keep the probability of dropped calls at lowest levels.

Fig. 9 Surface analysis for dropped calls rate between time value and the width of overlapping area for low velocity $(25 \mathrm{~km} / \mathrm{h})$

Moreover, results can be suitable for slow and medium mobility users up to $50 \mathrm{kmph}$. However, in fast mobility user, the situation can be more complicated and timer value require to be adaptive upon user speed and the width of overlapping area to avoid call dropping rates and reduce the ping-pong $\mathrm{HO}$ rates. The avoidance of ping-pong $\mathrm{HO}$ in real environment requires an accurate trade-off between timer value, width of overlapping area and the velocity of the mobile user. 


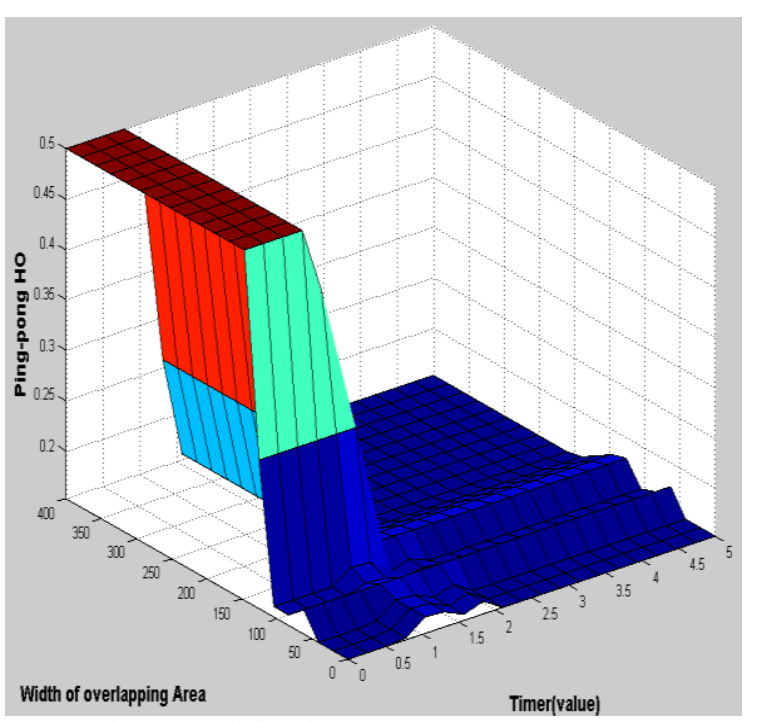

Fig. 10 Surface analysis for ping-pong HO between time value and the width of overlapping area for low velocity $(25 \mathrm{~km} / \mathrm{h})$

In high speed scenario explained in fig. 11, it is crucial to take to our consideration that the timer value should be selected to be less than 1.5 second for high speed users with respect to the width of the overlapping area. Timer values (higher than $1.5 \mathrm{sec}$ ) will increase the dropped calls rate. This can reduce the user experience and directly affect the connection. Narrow overlapping area in high speed movements can increase the probability of the dropped calls as it can be seen from figure 11.

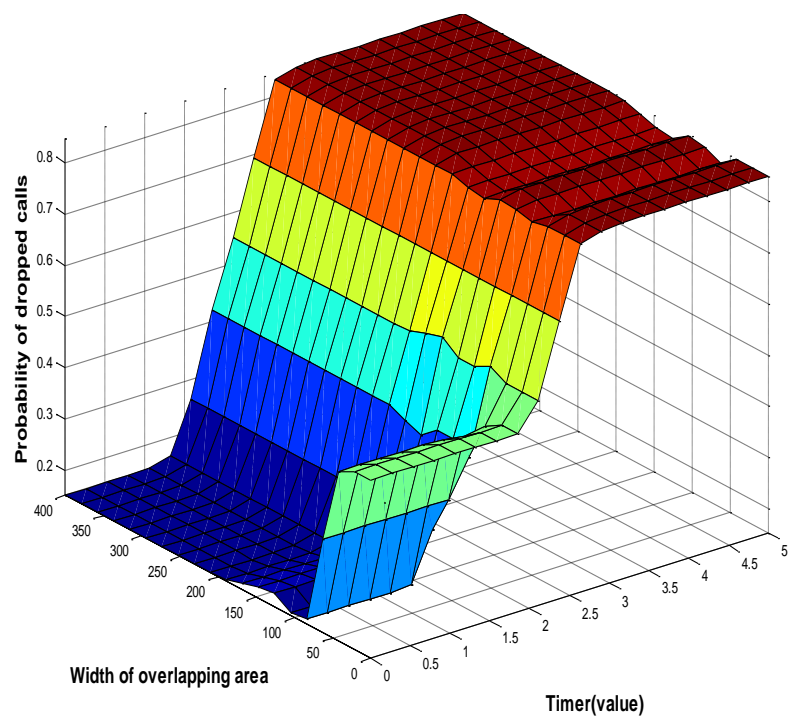

Fig. 11 Surface analysis for dropped calls rate between time value and the width of overlapping area for high velocity $(100 \mathrm{~km} / \mathrm{h})$

In high speed velocities, handover delay should be taken as a low value (less than $1 \mathrm{sec}$ based on the user speed). Fig. 12 indicates that the handover delay time decreases as the speed of UE increases. High mobility can cause higher handover failure probability, which seriously degrades the reliability of the user communication. Selecting a timer value based on the user velocity can decrease the probability of dropped calls rate.
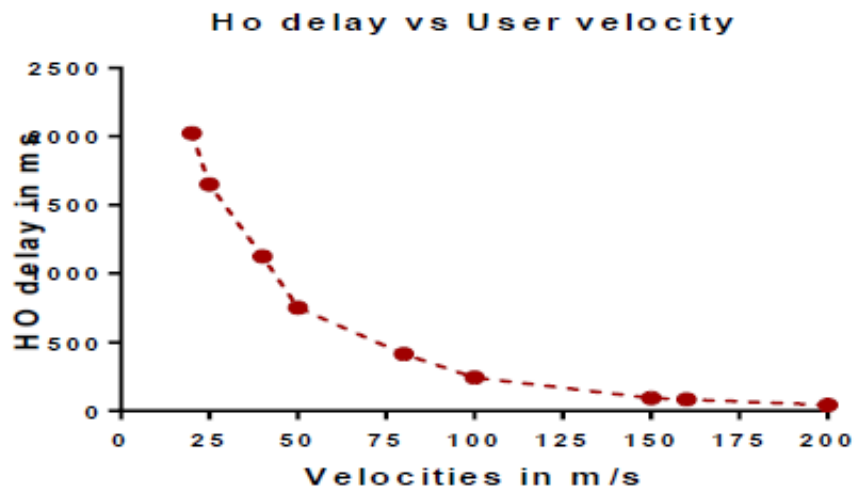

Fig. 12 The relationship between the user velocity in $\mathrm{m} / \mathrm{s}$ and the $\mathrm{HO}$ delay time in $\mathrm{ms}$

Another mean to keep the probability of dropped call rate and Ping-Pong $\mathrm{HO}$ rate at lowest level in high speed scenarios is to consider the width of overlapping area between neighboring cells. This can be achieved by adjusting the overlapping area according to the user velocity. It can been shown from figure 13 that the optimal overlapping area can be adjustable according to the user velocity (in the range of $8 \%-19 \%$ ). Overlapping area in figure 13 is taken as a percentage of the neighboring cells size. Selecting a proper overlapping area based on the user speed can increase the user experience and the quality of the handover.

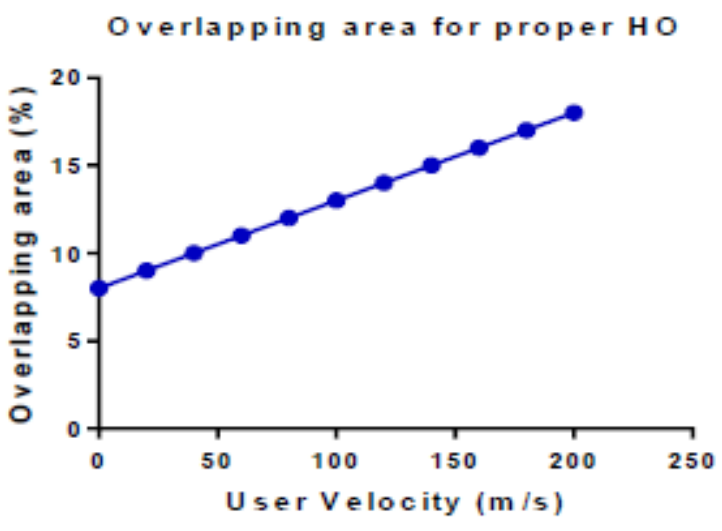

Fig. 13 The relationship between the user velocity in $\mathrm{m} / \mathrm{s}$ and the overlapping area

\section{CONCLUSIONS}

In this paper, the effects of overlapping area on the ping-pong $\mathrm{HO}$ in intra E-UTRA were studied. A novel ping-pong avoidance approach - based on adaptive 
timer value- to reduce the ping-pong HO in E-UTRA was presented. The presented scheme uses only timer value to delay the completion part only of the HO procedure as a trial to reduce the ping-pong $\mathrm{HO}$ rates and at the same time keep the probability of dropped calls at a low rate. The performance evaluation of the suggested algorithm was obtained using fuzzy logic technique. Results showed that the width of overlapping area play significant role in selecting the optimal timer value to reduce the ping-pong $\mathrm{HO}$ rates. For high speed velocity the timer value should be kept less than $1 \mathrm{sec}$ to prevent the probability of dropped calls and avoid the undesirable effects of ping-pong HO. Further work will consider the effects of overlapping area in heterogeneous networks.

\section{REFERENCES}

[1] J. Rodriguez, 'Radio Resource Management for Relayed Enhanced LTE Networks": A case based on centralized uplink. First Edition, LAP LAMBERT Academic Publishing, Denmark, 2011, P 367

[2] B. SINGH, "Hard handover performance evaluation through link drops," Signal Processing, Communications and Networking, ICSCN '07, International Conference, Feb 2007, pp. 459-463.

[3] H. Jihai and W. Bingyang, "Handover in the 3GPP long term evolution (LTE) systems," in Mobile Congress (GMC), 2010 Global, pp. 1-6.

[4] 3GPP TS 36.300: "Evolved Universal Terrestrial Radio Access (EUTRA); Overall description", Dec 2011.

[5] K. Ghanem , H. Al-radwan, A. Motermawy, A. Ahmad:" Reducing Ping-Pong Handover Effects In Intra E-UTRA Networks “ IEEE CSNDSP 2012,19-23, Poznan, Poland, July 2012.

[6] N. Benvenuto, F. Santucci,"A generalized least squares handover algorithm for wireless systems," IEEE VTC2002-Fall, pp. 15701574, Sept. 2002

[7] L. Wu, A. Sabbagh, K. Sandrasegaran, M. Elkashlan, " Performance Evaluation on Common Radio Resource Management Algorithms " Advanced Information Networking and Applications Workshops (WAINA), 2010 IEEE 24th International Conference on Advanced Information Networking Page(s): $491-495$

[8] K. LARS RASMUSSEN, "On Ping-Pong Effects in Linear Interference Cancellation for CDMA," in Proc. IEEE 6th ISSTA (New Jersey, USA), Sept. 2000.

[9] H. Jihai and W. Bingyang, "Handover in the 3GPP long term evolution (LTE) systems," in Mobile Congress (GMC), 2010 Global, pp. 1-6.

[10] S. Lal and D. Panwar, "Coverage Analysis of Handoff Algorithm with Adaptive Hysteresis Margin," Proc. 10th International Conference on Information Technology (ICIT 2007), Dec. 2007, pp. 133 - 138, doi: 10.1109/ICIT.2007.68

[11] T. Jansen, I Balan., J. Turk, I. Moerman, X. Ku, , and T. rner, "Handover Parameter Optimization in LTE Self-Organizing Networks," in Vehicular Technology Conference Fall (VTC 2010Fall), 2010 IEEE 72nd, pp. 1-5.

[12] A.Salkintzis K., M. Hammer, I. Tanaka, and C. Wong, "Voice Call Handover Mechanisms in Next-Generation 3GPP Systems," in IEEE Communications Magazine Motorola, Cisco, NTT DOCOMO, Nokia, and Siemens Networks, Feburary, 2009.

[13] G. Huaining, W. Xiangming , W. Zheng ,LuA Z. " HistoryBased Handover Prediction for LTE Systems " International Symposium on Computer Network and Multimedia Technology, 2009. CNMT 2009.978-1-4244-5272-9, pages 1-4

[14] D.Aziz, and R. Sigle, "Improvement of LTE handover performance through interference coordination," IEEE VTC Spring 2009, pp. 1-5.
[15] J., Jung, et al., "Mobility Prediction Handover Using User Mobility Patternand Guard Channel Assignment Scheme”, LNCS, Vol.3264, 2004

[16] A. Omri, R. Bouallegue, R. Hamila, and M. Hasna, "Channel Estimation for LTE Uplink System by Perceptron Neural Network," International Journal of Wireless \& Mobile Networks (IJWMN), vol. 2, pp. 155-165, 2010.

[17] D. Manoreet, et al., "Fuzzy Logic Based Handoff in Wireless Networks," Proceedings of IEEE VTC 2000 Spring Tokyo, Japan.

[18] Y. Chan, et. al. "Mobility Management Incorporating Fuzzy Logic for a Heterogeneous IP Environment," IEEE Communication Magazine, Dec. 2001.

[19] P. Chan M. L., R. E. Sheriff, Y. F. Hu, P. Conforto, C. Tocci, "Mobility Management Incorporating Fuzzy Logic for a Heterogeneous IP Environment," IEEE Communications Magazine, Dec. 2001. pp. 42-51

[20] M.S. Dang, et al., "Fuzzy Logic Based Handoff in Wireless Networks," Proc. VTC 2000 Spring, Tokyo, Japan, 15-18 May 2000, pp. 2375-79.

[21] L. Zadeli, , "Fuzzy Logic" IEEE J. Computer, Apr. 1988, pp. 83 93.

[22] M. Sugeno, and T. Yasukawa, "A Fuzzy-Based Approach to Qualitative Modeling," IEEE Transactions on Fuzzy Systems, Vol. 1, No. 1, Feb. 1993, pp. 7 - 31.

[23] D. Brubaker, "Fuzzy-logic system solves control problem," EDN Magazine, June 1992, pp. 121 - 127.

[24] T. ROSS, et al; Fuzzy Logic with Engineering Applications; McGraww-Hill Inc; New York(1995). 